\title{
Effects of Space and Scenery on Virtual Pet-Assisted Activity
}

\author{
Kenta Nakajima \\ Graduate School of Science and Engineering \\ Chuo University \\ Tokyo, Japan \\ E-mail: nakajima.kenta.lab@gmail.com
}

\author{
Mihoko Niitsuma \\ Department of Precision Mechanics \\ Chuo University \\ Tokyo, Japan \\ E-mail: niitsuma@mech.chuo-u.ac.jp
}

\begin{abstract}
In recent years, $15 \%$ of the working population have suffered from mental disorders. Such conditions cause low productivity, absence of diseases, and unemployment. In addition, the treatment of mental disorders takes a long time, so routine precautions are essential. One of the ways to prevent mental disorders is to achieve healing through interaction with animals. However, this method has not been widely accepted because of the difficulties in handling animals. To solve these problem, we introduced a virtual pet through a virtual reality technology for three-dimensional stereoscopic visualization as an alternative to animals. We named the system that prevents mental disorders by interacting with the virtual pet as virtual pet-assisted activity (VAA) and verified that this system gained short-term psychological and physiological benefits. However, it was unclear what causes the system to have positive effects on users. This study investigated whether space factors affect the psychological and physiological aspects of the user and whether the scenery factor affected the psychological and physiological aspects of the user and its interactions with the virtual pet. The experiment was divided into two experiments for the space factor and scenery factor. As a result, it was not affected by the space factor but was affected by the scenery factor. The findings reveal that the open scenery is more effective than the closed scenery in the VAA system.
\end{abstract}

\section{CCS CONCEPTS}

Human-centered computing, interaction design, interaction design process, and methods.

\section{KEYWORDS}

Virtual Reality (VR); Animal Assisted Activity (AAA); HumanRobot Interaction; Social Robotics

\section{ACM Reference format:}

Kenta Nakajima and Mihoko Niitsuma 2020. Effects of Space and Scenery on Virtual-Pet-Assisted Activity. In Proceedings of the 8th International Conference on Human-Agent Interaction, November 10-13, 2020, Virtual Event, Australia. (HAI'20), ACM, NY, NY, USA. 7 pages. https://doi.org/10.1145/3406499.3415083

Permission to make digital or hard copies of all or part of this work for personal or classroom use is granted without fee provided that copies are not made or distributed for profit or commercial advantage and that copies bear this notice and the full citation on the first page. Copyrights for components of this work owned by others than ACM must be honored. Abstracting with credit is permitted. To copy otherwise, or republish, post on servers or to redistribute to lists, requires prior specific permission and/or a fee. Request permissions from Permissions@acm.org.

HAI '20, November 10-13, 2020, Virtual Event, Australia.

(๑) 2020 Association of Computing Machinery

ACM ISBN 978-1-4503-8054-6/20/11_..\$15.00.

DOI: https://doi.org/10.1145/3406499.3415083

\section{Introduction}

In recent years, $15 \%$ of the working population have suffered from mental disorders. However, affected individuals are not properly treated in most countries, and one-third to half of them do not receive therapy. Such conditions cause low productivity, absence of diseases, and unemployment. In addition, the costs of mental ill-health can amount to more than $4 \%$ of the global GDP [1]. Therefore, the demand for measuring mental disorders is increasing. The treatment of mental disorders takes a long time, and the patient's productivity is reduced during treatment. To improve this condition, routine precautions are essential.

One of the ways to prevent mental disorders is to receive healing through interactions with animals, which include animalassisted therapy (AAT), which is the planned inclusion of an animal in a patient's treatment plan, and animal-assisted activity, which is the use of animals in recreational and visitation programs to help people with special needs [2]. Many studies on AAT and AAA have been conducted, and both activities have confirmed psychological, physiological, and social benefits [3] [4]. The cause of these benefits in AAT or AAA is not systematized, but relationships, such as bonds or affection between humans and animals, are important. However, to name some problems, animals need special training and care, hospital do not accept animals in sanitary, and some patients are allergic to animals.

To solve these problems, the robot-assisted activity (RAA) using a pet robot instead of animals has been researched. Pet robots are mechanical dolls that exist as objects in a real space. In addition, they can be physically touched, so the effects of helping to build a good relationship and direct healing effects are expected. Many studies on RAA have been conducted. For instance, the seal robot PARO [5] [6], which was approved by the Food and Drug Administration in America for its effectiveness in improving and preventing dementia, is well known. However, the degree of freedom in appearance and movement is low, and interactions with pets are limited to maintain the robot's balance. PARO was designed as a seal because users feel uncomfortable with the motion of robots when familiar animals are used as motifs.

Here, we explain the three benefits of AAT and AAA in the prevention of mental disorders: First, psychological benefits include the reduction of anxiety due to the existence of someone who does not deny, feeling of accomplishment of common goals, and improvement of morale. Second, physiological benefits include increased activities due to the motivation to exercise and reduction of blood pressure and cholesterol levels. Finally, the social benefit is that animals become a mediator between the user and others [7]. These benefits interact with one another to 
enhance the effect, so an environment providing all the benefits is recommended. It is difficult for pet robots with limited movements to directly provide physiological benefits or motivate users to exercise. To facilitate the acquisition of these benefits, it is desirable to induce exercise to interact with something that has a high degree of freedom of movement.

Therefore, we introduced a virtual pet using virtual reality (VR) technology for three-dimensional (3D) stereoscopic visualization as an alternative to animals or pet robots. 3D stereoscopic visualization enables depth perception by presenting separate images for the left and right eyes, taking binocular parallax into account. VR technology using 3D stereoscopic vision itself is a field that has been rapidly attracting attention in recent years, so sufficient knowledge has not been accumulated. We named the system that prevented mental disorders by interacting with a virtual pet as virtual pet-assisted activity (VAA) and verified that this system gained short-term psychological and physiological benefits [8].

The remainder of this paper is structured as follows. In Section 2, we describe the constitution of the VAA system, such as the virtual pet and VR space. In Section 3, we describe the study design. In Sections 4 and 5, we describe the experiments to investigate the effect of the space factor and scenery fator in VAA. Finally, in Section 6, we present the conclusions of this study.

\section{Application}

In this study, we developed a model of virtual pets, environment, and interaction based on the knowledge of the AAA In AAA, it is important that users and animals understand each other. However, it is not necessary to understand each other, so long as the user misunderstands that. Therefore, it is necessary for the user to think that the virtual pet has a heart and become familiar and interested in the virtual pet.

\subsection{System Software}

The VAA application is developed using the Unity3D Professional game engine. Assets which are Unity3D parts are adopted for the basic motion of CG models and virtual pets. The virtual pet model in the VAA system is the dog (dachshund) most often used in AAA (see Figure 1). Dogs have evolved independently because they have been companion animals since ancient times [9]. There is a lot of knowledge about dogs, and people can easily imagine and interact with it. In particular, dachshund is friendlier.

Next, the requirement for the VR space is that the interaction with the virtual pet is unobstructed and does not feel something is wrong. If the environment in the VR space is indoor, then the physical activity of the user and the virtual pet are limited. Therefore, the scenery in the VR space is grassland and blue sky. We named this space as a dog park (see Figure 2).

\section{$2.2 \quad$ System Hardware}

To enable intuitive interactions with the virtual pet, the scale of the VR space is matched with the real space. The position and rotation of the head are measured to present an image suitable for self-position. Furthermore, the hands used for the interaction with the virtual pet are measured. The head-mounted display (HMD) HTC VIVE Pro (see Figure 3) is used to measure the position and rotation and present the image. The controllers includ VIVE Pro with a vibration function. By gibing vibration to the hands, we aim to improve the sense of reality through pseudo-haptics. This system is constructed by matching the scale of the virtual pet and the objects in the VR space with real space.

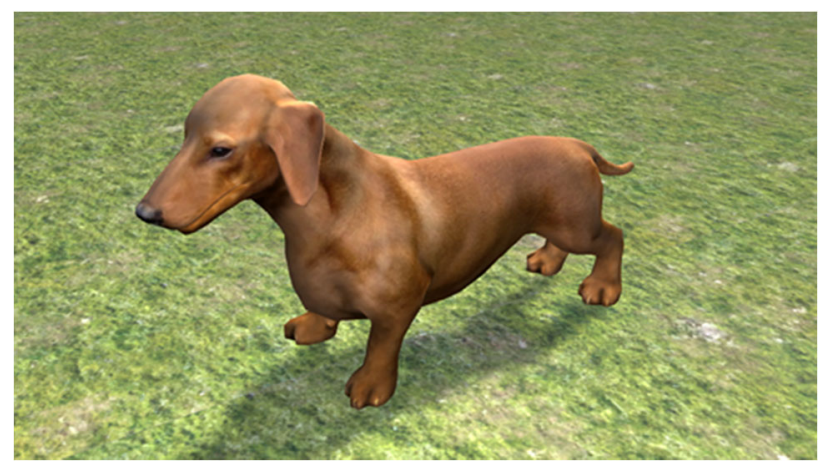

Figure 1: Appearance of the virtual pet. Assets which are Unity3D parts are adopted for the basic motion of CG models and virtual pets.

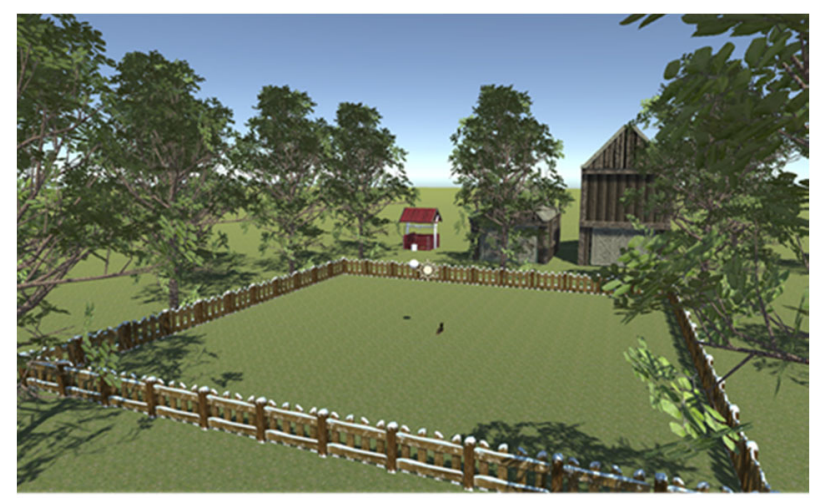

Figure 2: View of the dog park that consists of trees, sheds, fences, sun, grassland, and blue sky. The user interacts with the virtual pet in a $10 \mathbf{~ m}^{2}$ fenced area.

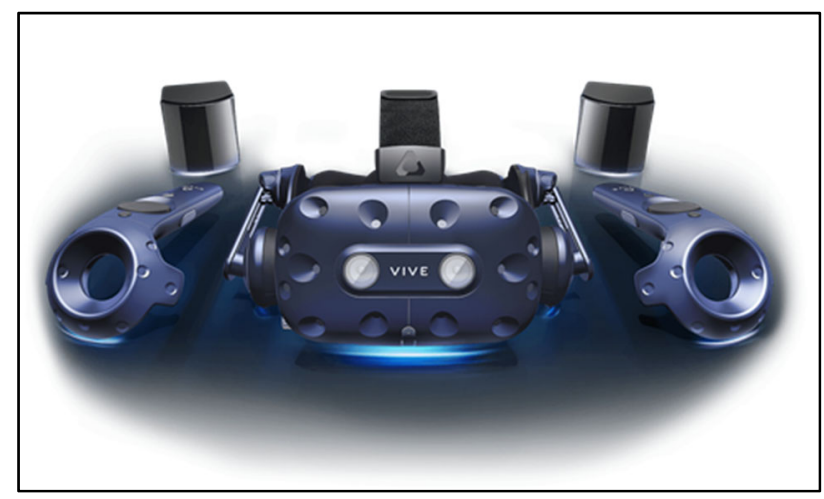

Figure 3: HTC VIVE Pro enables 3D stereoscopic visualization. It is used to measure the position and rotation and present the image. 


\subsection{Human-Virtual Pet Interaction}

The following describes the basic behavior of the virtual pet. People do not feel familiar when the virtual pet only behaves in the form of animals. The behavior of the virtual pet is designed so as not to break the mental model that people have for the model animals. When there is no action from the user, it feel uncomfortable if the virtual pet do not move, so the behavior of the virtual pet adopt a mechanism to act autonomously. The mechanism is Etho-Engine [10], which applies a dog's attachment behavior to a robot behavior model to realize a long-term and sustainable human-robot communication. In Etho-Engine, when the virtual pet is not called by the user, it is explored in the space. In addition, when the virtual pet is called by the user, it approached. It express its interest by always looking at the user's face. To look like a virtual pet that move leisurely next to the user, the walking speed is set to $2 \mathrm{~km} / \mathrm{h}$, which is half the general walking speed of humans. The running speed is set to $4 \mathrm{~km} / \mathrm{h}$, which is the general walking speed of humans and is not too fast.

In this study, the requirements for constructing a relationship between the virtual pet and users are as follows: the relationship should not break the mental model that users have, it should be familiar between them, and it should work on them. The interactions created based on these requirements are shown in Table 1. "Lay down" is the interaction where the virtual pet looks relaxed and gets attached to the user. "Play with the ball" is the bidirectional interaction where the virtual pet works with the user. Finally, "up the hill" is the interaction that induce the physical activity of the user.

\section{Study design}

The goal of this study is to prevent mental disorders by using this system and to suppress the decrease in productivity. We verified that the VAA system has psychological and physiological benefits. However, there were factors that could affect each effect other than the interaction with the virtual pet in this system, so it is unclear what caused the system to have positive effects on the user. In addition, unlike AAA, this system can easily set parameters or interaction types. To maximize the advantages of the system, it is important to investigate how they affect the interaction and the virtual pet and to use them for the system design. Each factor and its explanation are shown below.

Space factor: The VR space itself can affect the psychological and physiological aspects of the user by moving from the real space to the VR space.

Scenery factor: The open space can affect the psychological and physiological aspects of the user by moving the closed laboratory to the dog park in the VR space.
Exercise factor: The physical movement during the interaction with the virtual pet can affect the psychological and physiological aspects of the user.

Virtual pet factor: The cuteness of the virtual pet during the interaction can affect the psychological and physiological aspects of the user.

In this study, we investigate the space and scenery factors, among other factors, and reveal their effects.

In study 1, we investigated whether the VR space itself affected the psychological and physiological aspects of the user. We constructed a VR space that looked almost the same as the real space in which the experiment was performed, and the subjects performed tasks in both spaces. At that time, the psychological and physiological aspects of the subject were measured and discussed.

In study 2, we investigated whether the open space, such as the dog park in VR space, affected the psychological and physiological aspects of the user and the interaction with the virtual pet. The dog park was reconstructed as an open space, and the laboratory was constructed as a closed space in the VR space. The subjects interacted with the virtual pet in both spaces. At that time, the psychological and physiological aspects of the subject were measured and discussed..

\section{Study 1}

To investigate the effects of the VR space itself on the psychological and physiological aspects of users, we chose a controlled experiment in the real space and the VR space with the same visual. Therefore, the "scenery factor," "exercise factor," and "virtual pet factor" conditions were set the same in the two spaces. We set the scenery factor conditions to be the same as the real space by constructing the VR space that looked almost the same as the real space in which the experiment was performed. In addition, the "exercise factor" and "virtual pet factor" conditions were the same between the two spaces by setting the same task content.

\subsection{Materials and Procedure}

Our laboratory was chosen as the experiment location to secure the power supply to use the VR device. One section of our laboratory was used as the model of the VR space to be constructed. The created VR space was called the VR Lab. A preliminary experiment was conducted to determine whether there was a 3D difference between the constructed VR Lab and the laboratory in the real space and showed its usefulness as a space for experiments. Figure 4 shows the real space and the VR Lab.

Table1: Interaction with the virtual pet involves user's actions and the virtual pet's actions in response to it.

\begin{tabular}{|l|l|l|}
\hline \multicolumn{1}{|c|}{$\begin{array}{c}\text { Interactio } \\
\text { n }\end{array}$} & \multicolumn{1}{c|}{ User Action } & \multicolumn{1}{c|}{ Virtual Pet Action } \\
\hline Lay down & User keeps stroking the virtual pet. & Virtual pet lies down. \\
\hline $\begin{array}{l}\text { Play with the } \\
\text { ball }\end{array}$ & User throws a ball. & Virtual pet goes to get a ball. \\
\hline Up the hill & User guides the virtual pet to the athletics with controller. & Virtual pet goes up and down the athletics. \\
\hline
\end{tabular}


The subjects sat in the relaxation chair so as not to overload the body and performed the task of looking at each space. At that time, the movements of the neck and rotation of the chair were controlled so that the amount of physical activity among the subjects was the same. Currently, VR experience using HMD is not yet very popular, so the subjects had little or no VR experience, and the impression that the subjects received may be affected by freshness. To eliminate this, the same stimulus was given repeatedly and frequently according to the requirement of habituation [11]. In this experiment, one session was a minute task of looking, and four sessions were performed with a minute break. Furthermore, to eliminate the order effect, the subjects were divided into half, and the order of experiments was determined. The experiment was divided into 2 days. Eight men and women aged $21.6 \pm 1.11$ years (mean \pm SD) (range: $20-24$ years) participated in this experiment.

Next, the evaluation method is described. To measure the psychological effects, the Japanese version of the Positive and Negative Affect Schedule (PANAS) [12], [13], which comprises two mood scales, one that measures positive affect and the other that measures negative affect, proposed by Watson et al., was used. PANAS has 10 descriptors used for each PA scale and NA. The subjects were required to respond to 20 item tests using a six-point scale that ranges from slightly or not at all to extremely. In addition, to measure the physiological effects, a salivary amylase monitor that can measure the amount of salivary amylase [14], detected by saliva due to stress, was chosen. The salivary amylase activity is highly responsive, increases with unpleasant stimulation, and decreases with comfortable stimulation. Each evaluation was performed before the first session and after the final session.

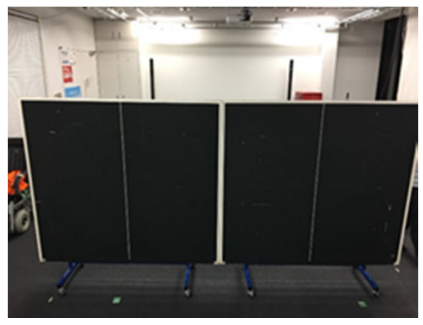

(a) Laboratory that exists in the actual space

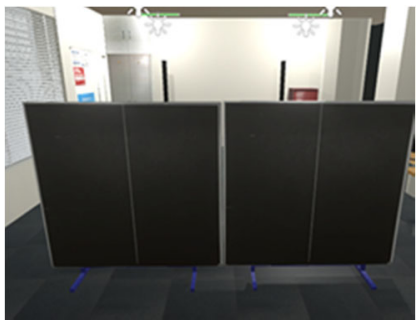

(b) Laboratory reproduced in the virtual space (VR Lab)
Figure 4: Space used for the experiment. VR Lab was reproduced in our laboratory with objects and textures.

\subsection{Results and Discussion}

The salivary amylase activity generally has a value of $10-100$ $\mathrm{kIU} / \mathrm{L}$. Data outside the range were excluded. The results of the mean salivary amylase activity in different conditions before and after the task is shown in Figure 5. No significant difference was confirmed before and after the task and between the VR space and the real space.

Next, the mean NA score and mean PA score (PANAS) before and after the task of each condition are shown in Figure 6. No significant difference was also confirmed before and after the task and between the VR space and the real space. This result implies that the VR space itself does not affect the psychological and physiological aspects of the user.

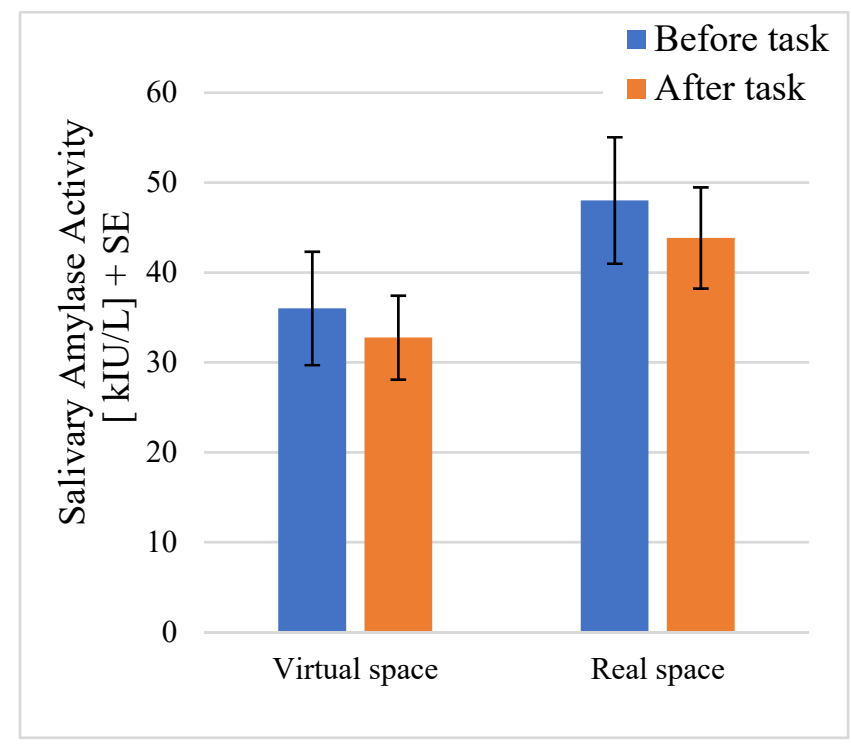

Figure 5: Salivary amylase activity was measured before and after the task in each condition. No significant difference was confirmed before and after the task and between the VR space and the real space.

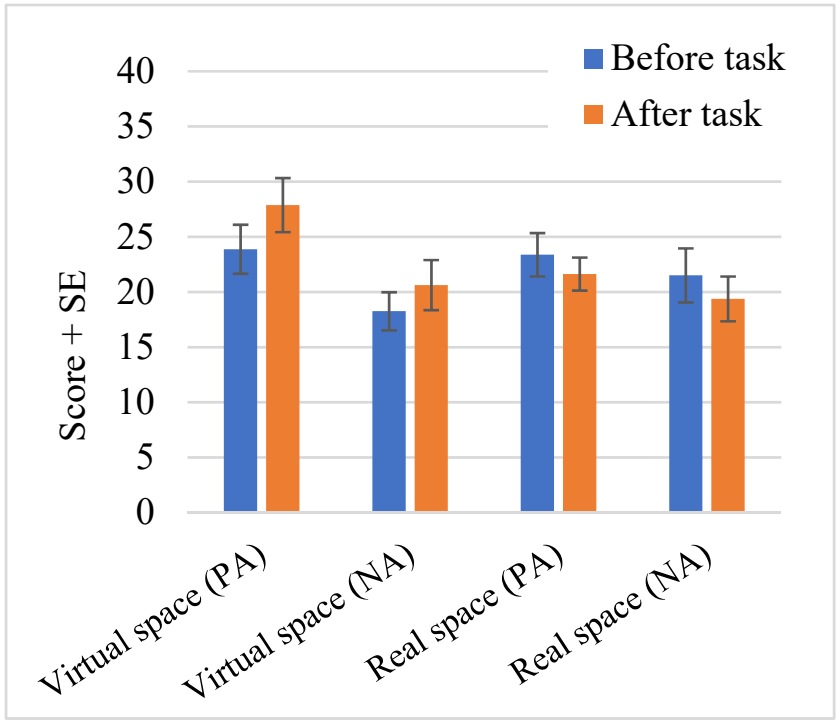

Figure 6: PANAS score was measured. No significant difference was confirmed before and after the task and between the VR space and the real space.

\section{$5 \quad$ Study 2}

In study 2, we investigated whether the scenery in the VR space affects the psychological and physiological aspects of the user and the interaction with the virtual pet. The controlled experiment was conducted in two VR spaces with different 
sceneries. The "exercise factor" and "virtual pet factor" conditions were the same between the two spaces by setting the same task content.

\subsection{Materials and Procedure}

In this experiment, the subjects interacted with the virtual pet in the two spaces with different sceneries. The first space was a dog park with many greens, which are said to have a positive effect on the psychological and physiological aspects of humans [15]. The second space was a VR lab constructed based on a laboratory that was a closed space paired with the dog park. A fence was newly added to the dog park so that it would be the same size as the VR lab, and the range of action of the virtual pet in each space was controlled to be the same.

In this experiment, tasks were decided based on the requirement of habituation. The interactions were that when the subject threw the ball, the virtual pet went to pick it up, and when the subject stroked the virtual pet, it lays down. The subject performed these interactions for four times and took a minute break. The task was set to 1 set, and four sets were performed. To eliminate the order effect, the subjects were divided into half, and the order of experiments was determined. The experiment was divided into 2 days. Eight men and women aged $20.5 \pm 1.50$ years (mean \pm SD) (range: 18-22 years) participated in this experiment.

We employed PANAS to measure the psychological effects and the salivary amylase activity to measure the physiological effects. These were performed before the first session and after the final session. In addition, the subjects answered the questionnaire using a five-point scale on the impression of the scenery, interactions, and the virtual pet after the final session. The question items are shown in Table 2 .

Table 2: Questionnaire on the impression of the scenery, interactions, and virtual pet.

\begin{tabular}{|l|l|}
\hline Number & \multicolumn{1}{|c|}{ Question } \\
\hline Q1 & Did you feel emotions from the dog? \\
\hline Q2 & Did you feel the dog was cute? \\
\hline Q3 & Did you feel the dog was smart? \\
\hline Q4 & Did you get along with the dog? \\
\hline Q5 & Did you feel like being with the dog? \\
\hline Q6 & Did you want to see the dog again? \\
\hline Q7 & Was the scenery comfortable? \\
\hline Q8 & Was it a proper place to play with the dog? \\
\hline Q9 & Did the dog move as you intended? \\
\hline Q10 & $\begin{array}{l}\text { How much did you enjoy moving in the virtual } \\
\text { space? }\end{array}$ \\
\hline
\end{tabular}

\subsection{Results and Discussion}

The salivary amylase activity generally has a value of $10-100$ $\mathrm{kIU} / \mathrm{L}$. Data outside the range were excluded. The results of the mean salivary amylase activity in different conditions before and after the task are shown in Figure 7. Under the dog park condition, the mean salivary amylase activity was significantly decreased $(t(6)=2.71, p=0.042)$ by interacting with the virtual pet but not in the VR Lab. This result implies that interacting with the virtual pet in an open space has physiological benefits.

Next, the mean NA score and mean PA score (PANAS) before and after the task of each condition are shown in Figure 8. The mean PA score was significantly increased $(\mathrm{t}(7)=2.65, \mathrm{p}=0.047)$ under the dog park condition from before to after the task. Furthermore, the mean NA score was significantly decreased $(t(7)=3.41, p=0.014)$. We also analyzed the mean PA and NA scores for the VR Lab condition. The mean NA score was significantly decreased $(\mathrm{t}(7)=4.73, \mathrm{p}=0.003)$ under the VR Lab condition. In addition, to investigate which space had a higher mean PA score and NA score differences before and after the task, the mean score in each condition was subtracted after the task from before the task. The differences in each score are shown in Figure 9. The mean PA difference was shown to be significantly higher $(t(7)=2.45, p=.042)$ under the dog park condition than the VR Lab condition. This result implies that interacting with the virtual pet in both spaces has psychological benefits, and an open space is more effective for obtaining them than a closed space.

The results of the questionnaire are shown in Figure 10. The scores of Q6, Q7, and Q8 were shown to be significantly higher (Q6: $\mathrm{t}(7)=2.52, \mathrm{p}=0.045 ; \mathrm{Q} 7 \mathrm{t}(7)=4.60, \mathrm{p}=0.003$; $\mathrm{Q} 8 ; \mathrm{t}(7)=3.20$, $\mathrm{p}=0.018)$ under the dog park condition than under the VR Lab condition. These results imply that the subjects feel stronger in the open space than the closed space as presented by their responses in the statements "I want to see the dog again," "This place was a proper place to play with the dog," and "this scenery was comfortable."

The following has been discussed for the salivary amylase activity, PANAS, and questionnaires. The open space was a more comfortable place and more proper place to play with dogs than the closed space. Furthermore, the subjects strongly felt that they wanted to see the dog again in an open space. From these results and the results of each evaluation, it is suggested that the scenery factor affects the interaction with the virtual pet. In addition, the interaction with the virtual pet in the open space, such as the dog park, contributes to the long-term relationship with the virtual pet and has more psychological and physiological effects due to the scenery itself or scenery that suits the situation. To obtain physiological benefits using the VAA system, it is necessary to set up an open space. Although psychological benefits are obtained regardless of the scenery factor, it is desirable to set up an open space to obtain more effects.

The following has been discussed for VAA in the future based on the findings. At the time of introduction of VAA, in order to build a relationship with the virtual pet and to be more effective, user interacts in an open space. Although, since psychological benefits are obtained even in a closed space like VR Lab, it may be useful for the design of a system that user does not get tired when used depending on the situation. 


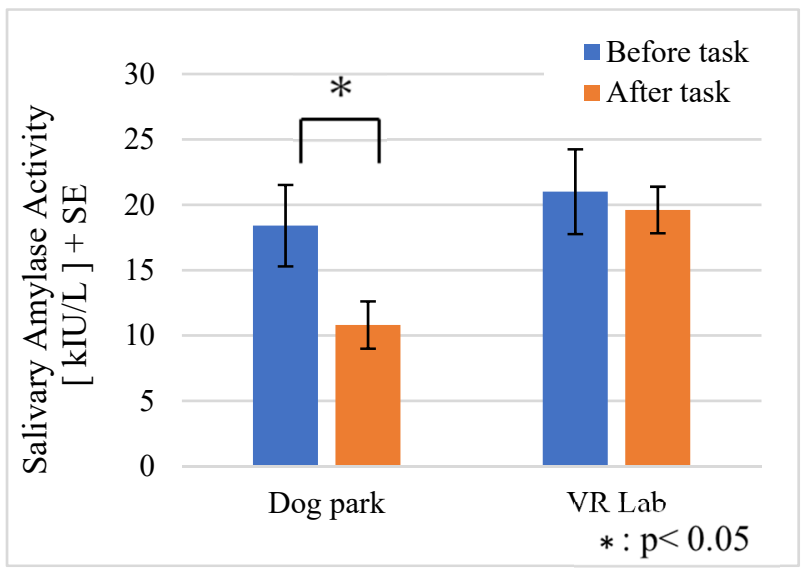

Figure 7: Salivary amylase activity was measured before task and after task in each condition. Under the dog park condition, the mean salivary amylase activity significantly decreased with the interaction on the virtual pet.

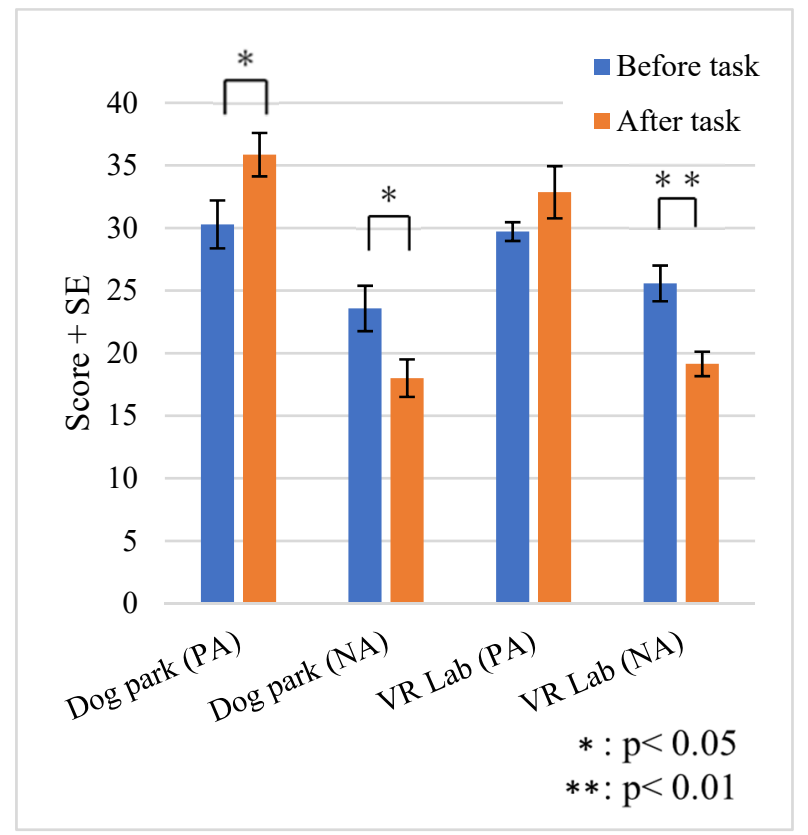

Figure 8: PANAS score was measured. The mean PA score was significantly increased under the dog park condition. Furthermore, the mean NA score was significantly decreased under both conditions.

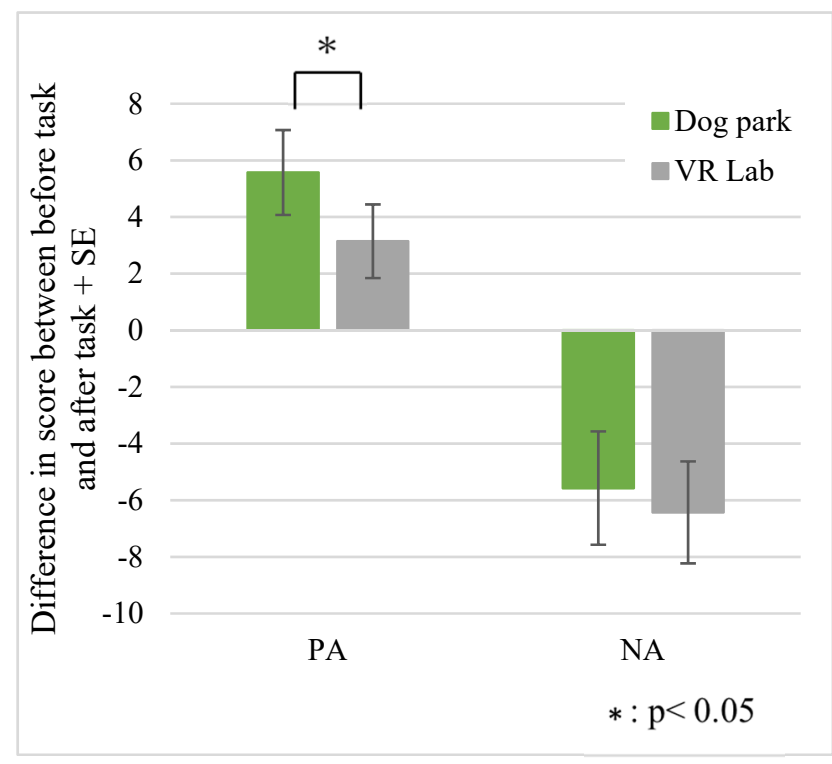

Figure 9: Difference of the mean score between before and after the task was calculated. The mean PA difference was shown to be significantly higher under the dog park condition.

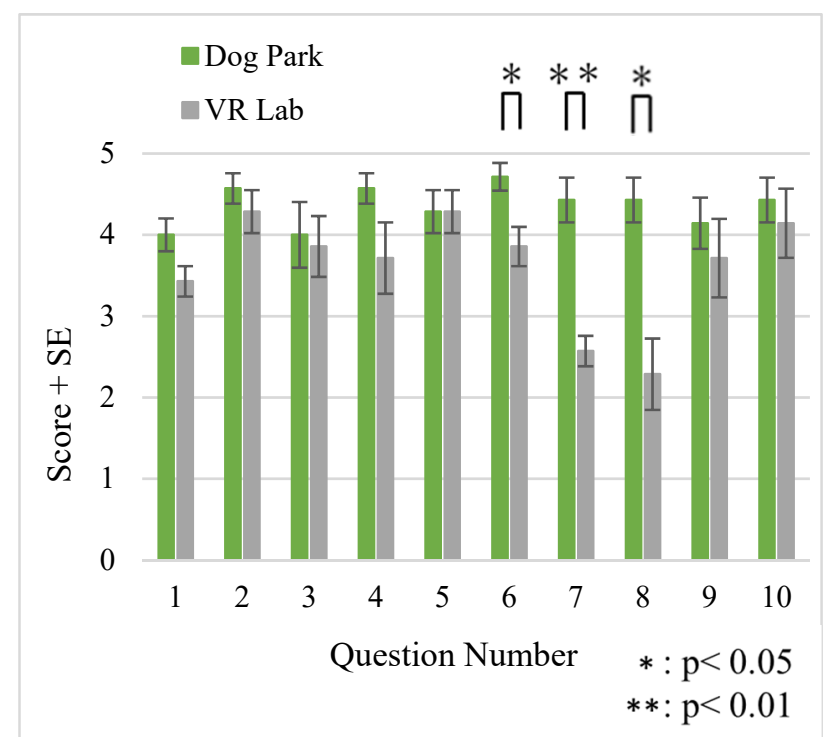

Figure 10: Questionnaire was conducted after the task. The scores of Q6, Q7 and Q8 were significantly higher under the dog park condition.

\section{Conclusion}

This study has verified whether the space and scenery factors of our VAA system affect the psychological and physiological aspects of users. First, to investigate the effect of the space factor, we performed a controlled experiment in which the task of looking was conducted in a VR space and real space. As a result, we could not find a significant difference. Next, we verified 
whether scenery in the VR space affects the interaction and psychological and physiological aspects of users. We also performed a controlled experiment in which the interaction with the virtual pet in open and closed spaces. The results show that the scenery factor affects the interaction with the virtual pet and psychological and physiological aspects of users. The physiological benefits were verified by interacting with the virtual pet only in the open space. Moreover, interacting with the virtual pet in both spaces has psychological benefits, and the open space is more effective for obtaining them than a closed space. The interactions in the open space contribute to the long-term relationship with the virtual pet. These results are probably attributed to the scenery itself or scenery that suits the situation.

Future work involves investigations of factors other than the ones investigated in this study and investigations of social effects, such as promotion of communication with others through interactions with the virtual pet in the VAA system. In addition, a system will be developed for long term, such as actual prevention. For example, to use the VAA system for a long term, it is necessary to maintain the user's interest in the system, so we will build a new virtual pet behavior model based on the knowledge of ethorobotics [16].

\section{REFERENCES}

[1] OECD, "Making Mental Health Count - OECD," [Online]. Available: https://www.oecd.org/els/healthsystems/Focus-on-Health-Making-Mental-HealthCount.pdf. [Accessed 3004 2020].

[2] P. Arkow, "What is AAT/AAA?," [Online]. Available: https://www.animaltherapy.net/what-is-aataa/. [Accessed 304 2020].

[3] P. W. Anderson, M. C. Reid , L. G. Jennings, "Pet ownership and risk factors for cardiovascular disease," The medical Journal of Australia, Volume 157, Issue 5, pp.298-301, 1992.

[4] E. Friedmann, H. A. Katcher, J. J. Lynch and A. S. Thomas, "Animal companions and one-year survival of patients after discharge from a coronary care unit," Public Health Rep, Volume95 Issue 4, p.307-312, 1980.

[5] R. Aminuddin, A. Sharkey, L. Levita, "Interaction With the Paro Robot May Reduce Psychophysiological Stress Responses," 2016 11th ACM/IEEE International Conference on Human-Robot Interaction (HRI), 2016.

[6] T. Shibata, "Development and spread of therapeutic medical robot, PARO: Innovation of nonpharmacological therapy for dementia and mental health," Journal of Information Processing and Management, 2017, Volume 60, Issue 4 ,pp217-228, 2017.

[7] A. H. Fine, Handbook on Animal-Assisted Therapy: Theoretical Foundations and Guidelines for Practice, Elsevier, 2011.

[8] K. Nakajima, J. Fukada , M. Niitsuma, "Effects of RealScale Body Motion on Virtual Pet Assisted Activity," 2019 JSME Conference on Robotics and Mechatronics, 2019.

[9] D. W. Paxton, "A Case for a Naturalistic Perspective," Anthrozoos ,13,pp5-8, 2000.
[10] S. Takahashi, G. Marta, P. Korondi, H. Hashimoto , M. Niitsuma, "Leading a Person Using Ethologically Inspired Autonomous Robot Behavior," 10th ACM/IEEE International Conference on Human-Robot Interaction (2015) pp. 87 - 88., 2015.

[11] C. H. Rankin, T. Abrams, R. J. Barry, e. a. , "Habituation revisited: An updated and revised description of the behavioral characteristics of habituation," Neurobiology of Learning and Memory, volume 92, Issue 2, pp135-138, 2009.

[12] D. Watson, L. Clark, A. Tellegen, "Development and validation of brief measures of positive and negative affect: the PANAS scales," Journal of Personality and Social Psychology, 54 (1988) pp. 1063 - 1070., 1988.

[13] S. Sato, A. Yasuda, "Development of the Japanese version of Positive and Negative Affect Schedule (PANAS) scales," Japan Society of Personality Psychology, volume 9, issue 2, pp.138-139, 2001.

[14] M. Yamaguchi, M. Kanemaru, T. Kanemori, Y. Mizuno , H. Yoshida, "Salivary amylase activity monitor used for stress evaluation," Proceedings of the 25th Annual International Conference of the IEEE EMBS, Cancun, 2003, pp. 3075-3078 Vol.4, 2003.

[15] M. M. Hansen, R. Jones , K. Tocchini, "Shinrin-Yoku (Forest Bathing) and Nature Therapy: A State-of-the-Art Review," International Journal of Environmental Research and Public Health, 2017.

[16] H. Kanai , M. Niitsuma, "Update of Human-Robot Relationship Based on Ethologically Inspired HumanRobot Communication History," 25th IEEE International Symposium on Robot and Human Interactive Communication (RO-MAN), 2016. 